\title{
Locking of domain walls and quadratic frequency combs in doubly resonant optical parametric oscillators
}

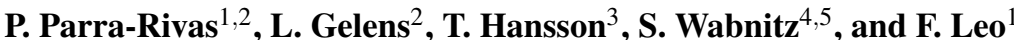 \\ 1. OPERA-photonics, Universit libre de Bruxelles, 50 Avenue F. D. Roosevelt, CP 194/5, B-1050 Bruxelles, Belgium \\ 2. Laboratory of Dynamics in Biological Systems, KU Leuven Department of Cellular and Molecular Medicine, University of Leuven, B-3000 \\ Leuven, Belgium \\ 3. Department of Physics, Chemistry and Biology, Linkping University, SE-581 83 Linkping, Sweden \\ 4. Dipartimento di Ingegneria dell'Informazione, Elettronica e Telecomunicazioni, Sapienza University of Rome, Via Eudossiana 18, 00184 \\ Rome, Italy \\ 5. Istituto Nazionale di Ottica del CNR, Via Campi Flegrei 34, I-80078 Pozzuoli (NA), Italy
}

The formation of frequency combs (FCs) in high- $Q$ microresonators with Kerr type of nonlinearity has attracted a lot of attention in the past decade [1]. Recently it has been shown that FCs can be also generated in dissipative dispersive cavities with quadratic nonlinearities [2,3], opening a new possibility of generating combs in previously unattainable spectral regions. Previous work has shown that modulational instability (MI) induces pattern and FC formation in degenerate optical parametric oscillators (OPOs) [4]. However, the existence of dissipative solitons or localized structures (LSs) is still unclear. In this work we present the locking of domain walls (DWs) as an alternative mechanism to MI for the formation of LSs and FCs. DWs have been widely studied in the context of Kerr cavities and diffractive OPO cavities [5,6]. Here we show that similar structures can arise in dispersive quadratic cavities. To illustrate this, we consider a dispersive cavity with a quadratic medium phase matched for degenerate $\mathrm{OPO}$ and driven by the field $B_{i n}$ at the frequency $2 \omega_{0}$ in a doubly resonant configuration. The formation of dissipative structures in this type of cavity can be described by an infinite map for the slowly varying electric field envelopes $A_{m}$, and $B_{m}$, that are centered at frequency $\omega_{0}$ and $2 \omega_{0}$, respectively [4], where $m$ indicates the cavity round-trip number. With this map one may numerically explore the dynamics of the system. For example,

(a) $\quad \mathrm{LS}_{1} \quad \mathrm{LS}_{2} \mathrm{LS}_{3} \quad \mathrm{LS}_{4} \quad \mathrm{LS}_{5} \quad \mathrm{LS}_{6} \mathrm{LS}_{7}$

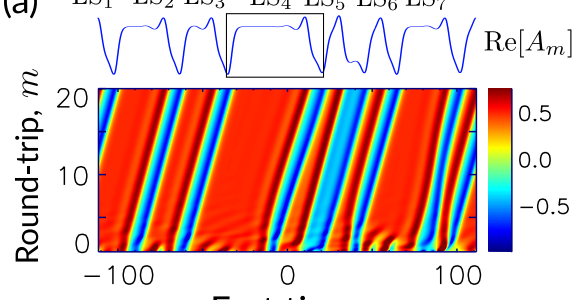

Fast time, $\tau$ (b)

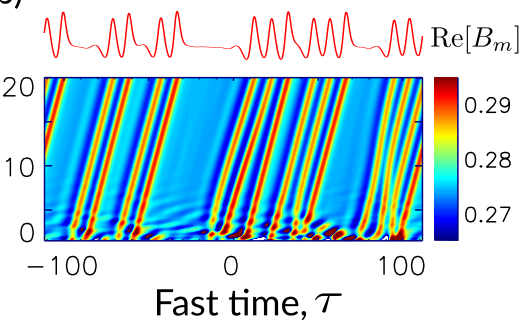

(c)

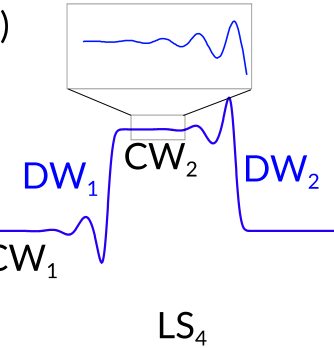

Figure 1: Panels (a) and (b) show the round-trip evolution of an initial noisy background [bottom], and the formation of a disordered stationary state composed of seven LSs [top]. Panel (c) shows how $\mathrm{LS}_{4}$ is formed through the locking of two DWs. To simulate the infinite map we use the same parameters as in Ref. [4].

Fig. 1(a)-(b) show the formation of a dissipative structure, after a sufficient number of round-trips $\left(\mathrm{m}=2 \cdot 10^{4}\right)$, from an initial noisy background. We see that i) both fields are phase locked and drift at the same velocity; ii) for the $A$ field one can identify a sequence of DWs connecting two different, and coexisting, CW states, that form a disordered structure. Furthermore, the latter is composed of a sequence of LSs of different widths. One of them, $\mathrm{LS}_{4}$, is plotted in panel (c). We demonstrate that this type of states can be described by a single non-local parametrically forced Ginzburg-Landau model for $A$, that can be derived by adiabatic elimination of $B$ [8]. In this context, the $B$ field is dynamically slaved to $A$. Using the last model, one may describe the formation of such structures through the interaction of the DWs. If oscillations are present in the DWs' profiles [see close-up view in (c)], then locking occurs, and LSs are formed, as described in [9]. In the frequency domain, these LSs correspond to coherent FCs formed around both $\omega_{0}$ and $2 \omega_{0}$.

\section{References}

[1] P. DelHaye, et al., Nature 450, 12141217 (2007).

[2] I. Ricciardi, et al., Phys. Rev. A 91, 063839 (2015).

[3] F. Leo, et al., Phys. Rev. Lett. 116, 033901 (2016).

[4] S. Mosca, et al., Phys. Rev. Lett. 121, 093903 (2018).

[5] V. E. Lobanov, et al., Opt. Express 23, 7713 (2015).

[6] P. Parra-Rivas, et al., Opt. Lett. 41, 2402 (2016).

[7] G.-L. Oppo, A. J. Scroggie, and W. J. Firth, J. Opt. B: Quantum Semiclass. Opt. 1133138 (1999).

[8] P. Parra-Rivas, et al. (submitted).

[9] P. Coullet, C. Elphick, and D. Repaux, Phys. Rev. Lett. 58, 431 (1987). 